Histoire de l'éducation

$119 \mid 2008$

Varia

WATTS (Ruth), Women in Science. A social and cultural history

Londres, New-York : Routledge, 2007, IX-300 p.

Frédéric Graber

\title{
OpenEdition
}

Journals

Édition électronique

URL : https://journals.openedition.org/histoire-education/1845

DOI : $10.4000 /$ histoire-education. 1845

ISSN : 2102-5452

Éditeur

ENS Éditions

Édition imprimée

Date de publication : 1 juillet 2008

Pagination : 89-91

ISBN : 978-2-7342-1124-2

ISSN : 0221-6280

Référence électronique

Frédéric Graber, «WATTS (Ruth), Women in Science. A social and cultural history », Histoire de l'éducation [En ligne], 119| 2008, mis en ligne le 20 mai 2009, consulté le 20 mai 2021. URL : http://

journals.openedition.org/histoire-education/1845; DOI : https://doi.org/10.4000/histoire-education. 1845

Ce document a été généré automatiquement le 20 mai 2021.

(c) Tous droits réservés 


\section{WATTS (Ruth), Women in Science. A social and cultural history}

Londres, New-York : Routledge, 2007, IX-300 p.

Frédéric Graber

\section{RÉFÉRENCE}

WATTS (Ruth), Women in Science. A social and cultural history, Londres, New-York :

Routledge, 2007, IX-300 p.

1 Ruth Watts, professeur d'histoire de l'éducation à l'université de Birmingham, propose ici une histoire de la place des femmes dans les sciences depuis l'Antiquité jusqu'à la Seconde Guerre mondiale, plus particulièrement en Grande-Bretagne et, surtout, en Angleterre. L'originalité de cet ouvrage est de croiser l'histoire des sciences, l'histoire de l'éducation et l'histoire des femmes (ou, plus généralement, les gender studies), à la fois pour remédier à une relative faiblesse des études sur les sciences et le genre en histoire de l'éducation et pour mieux articuler la participation des femmes aux pratiques savantes avec les problèmes de l'enseignement. Il s'agit de saisir, à chaque époque, ce qui constitue un savoir et la façon dont il est produit, pour comprendre comment se posent à la fois la question de l'éducation aux sciences et celle de l'accès des femmes à ces dernières. $R$. Watts cherche à tenir ensemble l'évolution des sciences et de ce qui est valorisé dans les savoirs avec la manière dont certaines personnes sont privilégiées ou marginalisées dans la production et la diffusion de ces savoirs. Au-delà de la seule question du genre, elle s'inscrit donc dans une tradition de réflexion sur les inégalités (les Anglo-Saxons utilisent plutôt ici le concept de power), avec, en particulier, une attention soutenue aux différences de classe sociale.

2 L'ouvrage se présente donc à la fois comme une synthèse et comme un travail de mise en relation d'historiographies assez différentes. Il faut mesurer la difficulté de l'entreprise pour rendre pleinement justice à ce livre ambitieux. Les champs que $R$. Watts entreprend de synthétiser et de croiser ici sont considérables, couvrant des 
périodes, des objets et des questionnements très nombreux. Il est donc inévitable que le lecteur regrette l'absence de tel ou tel auteur ou courant de recherche, mais il faut reconnaître que le résultat obtenu est remarquable, compte tenu des dimensions de l'étude. R. Watts propose une histoire panoramique, couvrant les grandes transformations des sciences et de l'éducation scientifique depuis l'Antiquité jusqu'au milieu du XXe siècle, en moins de deux cents pages. Le risque était donc grand de simplifier à l'extrême tant les objets et les pratiques scientifiques que ceux de l'éducation et d'aboutir à une marche forcée à travers les siècles. Seul, le deuxième chapitre, couvrant la période du Ve au XVIe siècle (p. 18-34), tombe peut-être sous cette critique, présentant un niveau de généralité et des sauts chronologiques qui font douter de son utilité, même si l'auteur est convaincue de l'importance des idées philosophiques et savantes de l'Antiquité, en particulier concernant le genre, pour la suite de l'histoire. Toujours est-il qu'après ces premières incertitudes, l'ouvrage parvient à trouver un équilibre intéressant : plutôt que de prétendre couvrir toutes les sciences et tous les contextes d'éducation, $R$. Watts choisit de se focaliser sur un certain nombre de situations, de lieux, de personnes et de réseaux, qui permettent plus particulièrement de comprendre les interactions qu'elle cherche à saisir entre sciences, genre et éducation. Le livre est ainsi construit autour d'une série de figures individuelles, choix qui entend rendre visibles les femmes en sciences, alors qu'elles ont souvent été effacées de la mémoire de leurs contemporains comme de celle des historiens. Mais R. Watts procède à bien davantage qu'une redécouverte (nécessaire, certes, mais parfois hagiographique) de la place des femmes dans les sciences, elle replace chacune de ces femmes dans un contexte suffisamment détaillé pour que le lecteur mesure les contraintes particulières qui ont pesé sur chacun de ces parcours. Plutôt que d'écrire une histoire générale, il s'agit donc plutôt de saisir la diversité des expériences des femmes dans le champ scientifique et le rôle des circonstances (religieuses, politiques, économiques, etc.) : c'est une histoire de fenêtres qui s'ouvrent et se ferment, d'occasions toujours localisées et qui, jusqu'au milieu du XXe siècle, ne semblent jamais vouloir se généraliser tout à fait.

3 Si aucun modèle de la situation des femmes dans le champ des sciences ne se dégage de cette lecture, R. Watts parvient toutefois à un certain nombre de conclusions générales, dont on reprendra ici quelques éléments. Si elle insiste tant sur les types de savoirs qui sont valorisés selon les époques, c'est que les femmes parviennent à s'impliquer plus facilement dans des pratiques ou des champs disciplinaires qui sont peu considérés socialement. La visibilité et le succès tiennent beaucoup aussi au niveau social de ces femmes. On peut s'étonner, d'ailleurs, que R. Watts n'ait pas porté plus d'attention aux techniciennes (sauf en médecine), aux petites mains des laboratoires, dans la mesure où les techniciens sont par excellence les invisibles dans les sciences. Il est vrai que ces parcours-là sont certainement très difficiles à reconstituer. Les femmes sont généralement plus présentes dans les sciences comme traductrices (pensons à Clémence Royer traduisant Darwin), enseignantes, passeuses de savoirs, que comme créatrices, mais, contre une historiographie centrée sur les héros de la science, il convient justement de revaloriser ces fonctions, en particulier celles d'enseignante et d'auteur de livres scientifiques (parfois spécialement destinés aux jeunes filles).

4 Le lieu apparaît comme un paramètre essentiel. Certaines institutions sont en effet plus ouvertes aux femmes, mais c'est surtout le cadre familial qui joue : c'est là, auprès d'un parent savant, ouvert aux sciences ou au moins à l'éducation des filles, que se joue la plus grande partie de la formation scientifique des femmes; en effet, si les cours de 
sciences se multiplient dans les écoles à partir de la fin du XIXe siècle, les savoirs destinés aux filles continuent à être déterminés par des stéréotypes de genre et se limitent souvent à des sciences pratiques et domestiques. Au-delà de la famille, ce sont les réseaux et les milieux qui sont déterminants, en particulier, à partir du XVIIIe siècle, ceux qui sont influencés par Joseph Priestley, les communautés religieuses radicales et/ou tolérantes, les groupes comme les Unitariens (auxquels R. Watts avait déjà consacré un ouvrage Gender, Power and the Unitarians in England, 1760-1860, Londres, Longman, 1999) ou les Quakers, qui ont très tôt envisagé l'éducation des filles comme celle des garçons, avec un intérêt remarquable pour les sciences. Enfin, l'ouvrage porte une grande attention au champ de la santé, qui offre une série de rôles traditionnellement féminins dont les femmes sont progressivement écartées avec la scientifisation de la médecine et la mainmise sur celle-ci d'une université longtemps exclusivement masculine (la partie la plus connue de ce phénomène étant la masculinisation de l'obstétrique et de la gynécologie). R. Watts décrit non seulement comment les femmes vont parvenir, non sans difficultés bien entendu, à s'imposer progressivement à partir du milieu du XIXe siècle dans cette médecine savante, mais plus généralement comment se créent en médecine de très nombreux emplois (infirmières, sages-femmes, etc.) réclamant des formations spécifiques, qui vont en retour stimuler la création de classes de sciences et l'emploi d'enseignantes de sciences.

\section{AUTEURS}

FRÉDÉRIC GRABER 